\title{
Ultrathin (1×2)-Sn layer on GaAs(100) and InAs(100) substrates: A catalyst for removal of amorphous surface oxides
}

\author{
P. Laukkanen, ${ }^{1,2, a)}$ M. P. J. Punkkinen, ${ }^{1,3}$ J. Lång, ${ }^{1}$ M. Tuominen, ${ }^{1}$ M. Kuzmin, ${ }^{1,4}$ \\ V. Tuominen, ${ }^{1}$ J. Dahl, ${ }^{1}$ J. Adell, ${ }^{5}$ J. Sadowski, ${ }^{5,6}{ }^{2}$ J. Kanski, ${ }^{7}$ V. Polojärvi, ${ }^{2}{ }^{2}$. Pakarinen, ${ }^{8}$ \\ K. Kokko, ${ }^{1}$ M. Guina, ${ }^{2}$ M. Pessa, ${ }^{2}$ and I. J. Väyrynen ${ }^{1}$ \\ ${ }^{1}$ Department of Physics and Astronomy, University of Turku, FI-20014 Turku, Finland and Turku University \\ Centre for Materials and Surfaces (MatSurf), Turku, Finland \\ ${ }^{2}$ Optoelectronic Research Centre, Tampere University of Technology, FI-33101 Tampere, Finland \\ ${ }^{3}$ Department of Materials Science and Engineering, Applied Materials Physics, Royal Institute of Technology, \\ SE-10044 Stockholm, Sweden \\ ${ }^{4}$ Ioffe Physical-Technical Institute of the Russian Academy of Sciences, St. Petersburg 194021, \\ Russian Federation \\ ${ }^{5}$ MAX-lab, Lund University, SE-221 00 Lund, Sweden \\ ${ }^{6}$ Institute of Physics, Polish Academy of Sciences, al. Lotnikow 32/46, 02-668 Warszawa, Poland \\ ${ }^{7}$ Department of Applied Physics, Chalmers University of Technology, SE-41296 Göteborg, Sweden \\ ${ }^{8}$ VTT Technical Research Center of Finland, FIN-02044 Espoo, Finland
}

(Received 15 December 2010; accepted 13 May 2011; published online 10 June 2011)

\begin{abstract}
Amorphous surface oxides of III-V semiconductors are harmful in many contexts of device development. Using low-energy electron diffraction and photoelectron spectroscopy, we demonstrate that surface oxides formed at $\mathrm{Sn}$-capped $\mathrm{GaAs}(100)$ and $\operatorname{InAs}(100)$ surfaces in air are effectively removed by heating. This Sn-mediated oxide desorption procedure results in the initial well-defined Sn-stabilized $(1 \times 2)$ surface even for samples exposed to air for a prolonged time. Based on ab initio calculations we propose that the phenomenon is due to indirect and direct effects of Sn. The Sn-induced surface composition weakens oxygen adsorption. (C) 2011 American Institute of Physics. [doi:10.1063/1.3596702]
\end{abstract}

Amorphous (or polycrystalline) oxide layers are easily formed at the surfaces of III-V semiconductors exposed to oxygen. The formation of such structurally and electronically poor III-V oxide layers is detrimental for the development of many types of devices. Detrimental effects include surface Fermi-level pinning in the III-V channel of metal-oxidesemiconductor field-effect transistors (MOSFETs), increased surface recombination, barrier formation within metalsemiconductor contacts, and surface contamination affecting regrowth processes. The first example is probably the best known; the formation of amorphous surface oxides at the interface between a III-V semiconductor and an insulator causes the Fermi level to pin via defect states, thus rendering the development of III-V channels difficult for practical MOSFETs. A significant effort has therefore been made to understand how III-V oxides can be removed (or their formation prevented) during the fabrication of insulator-III-V junctions. ${ }^{1-12}$ However, it is still unclear whether it is possible to avoid the oxygen reaction during interface growth, and there is still much room to improve insulator-III-V interfaces and our overall understanding of III-V oxidation. Simultaneously, an increasing number of studies ${ }^{5,13-19}$ has revealed the importance of atomic-scale information and of the processing of semiconductor surfaces, so-called surface engineering, for controlling the properties of the semiconductor interfaces. These studies have shown that a properly chosen thin adsorbate layer with a well-defined structure significantly improves the properties of oxide-semiconductor interfaces for various types of semiconductor devices.

In this letter, we report an interesting effect of Sncapping on the desorption properties of amorphous oxides of

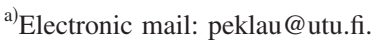

GaAs(100) and InAs(100) surfaces. In particular, we have found that the initial Sn-stabilized $(1 \times 2)$-ordered GaAs and InAs surfaces, comprising 0.5-1.0 monolayer (ML) of Sn, can be restored even after a week's exposure to air; in other words, the native surface oxides formed can be efficiently removed by heating the substrates in a vacuum. This property may be useful not only for the development of the longsought III-V MOSFETs but also for example for the development of Ohmic contacts and re-growth applications.

Deposition of $0.5-1 \mathrm{ML}$ of $\mathrm{Sn}$ and postheating $\left(500{ }^{\circ} \mathrm{C}\right.$ for $\mathrm{GaAs}$ and $300{ }^{\circ} \mathrm{C}$ for InAs) produced clear low-energy electron diffraction (LEED) patterns. Details of the experiments have been described recently. ${ }^{20}$ The reference (without Sn) and Sn-covered substrates were exposed to air in an open loading chamber and in a separate air-filled cabinet. Photoemission measurements were performed at the MAX-laboratory beamline 41 (Sweden), using a synchrotron source and at the home laboratory, using an $\mathrm{Mg} K_{\alpha}(1253.6$ $\mathrm{eV}) \mathrm{x}$-ray source. Details of our room temperature measurements can be found elsewhere. ${ }^{21-23}$

The photoemission and LEED measurements for the samples exposed to air are summarized in Fig. 1. Figure 1(a) shows a clear difference in $\mathrm{Ga} 3 d$ emissions between the $\operatorname{GaAs}(1 \times 2)$-Sn and the pure GaAs- $c(8 \times 2)$ surfaces after air exposure and postheating. The Ga $3 d$ of the air-exposed GaAs- $c(8 \times 2)$ is broadened due to Ga oxide emission, and shows a band-bending-related energy shift as compared to the clean GaAs- $c(8 \times 2)$. This observation is consistent with the appearance of the $\mathrm{O} 1 s$ emission [Fig. 1(b)]. LEED measurements for the air-exposed GaAs- $c(8 \times 2)$ revealed a poor $(1 \times 1)$ pattern, which was not improved after prolonged heating. In contrast, the $\mathrm{Ga} 3 d$ emission from the heated GaAs $(1 \times 2)$-Sn surfaces does not present any oxide-related 

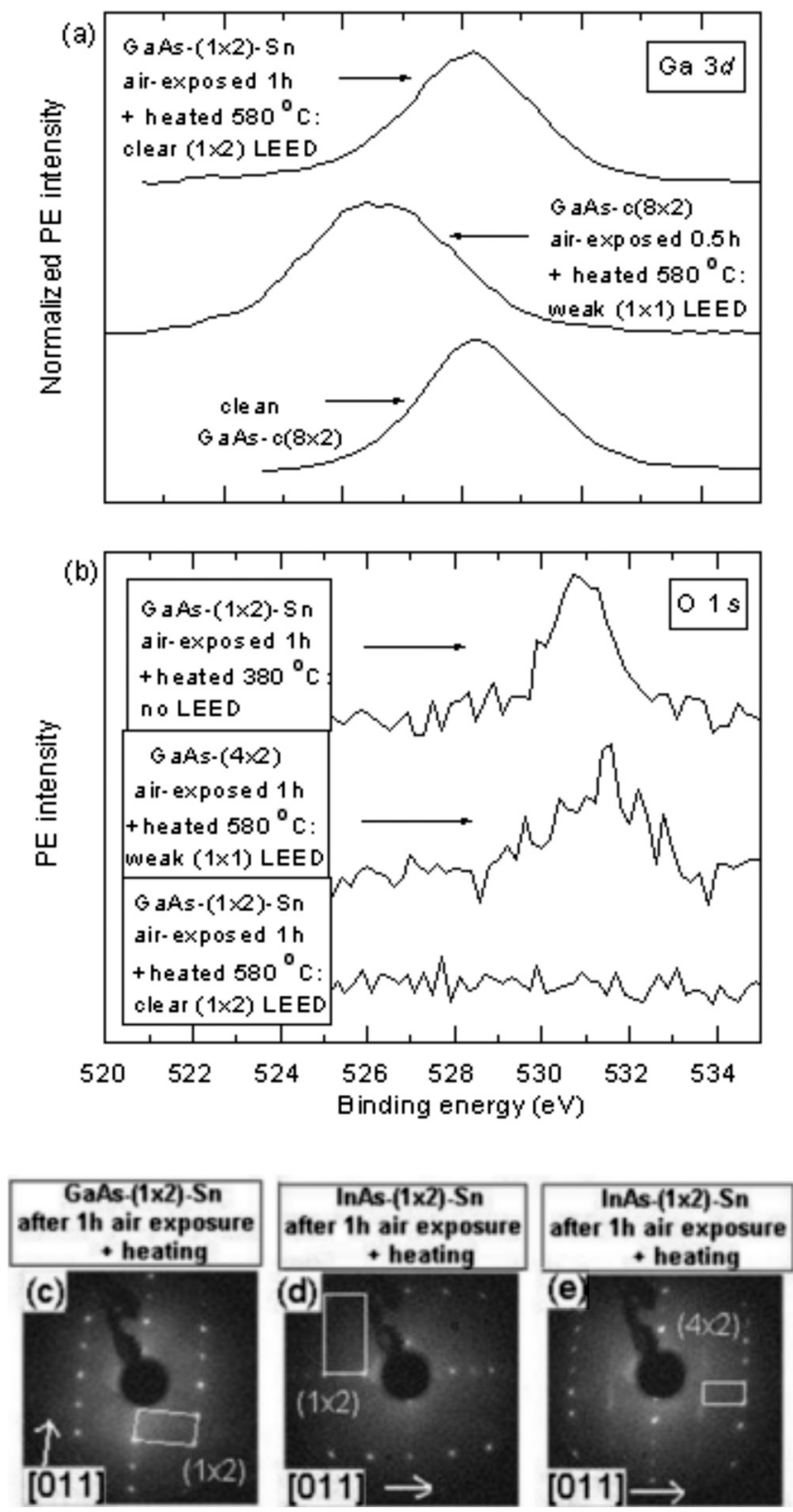

FIG. 1. (a) Ga $3 d$ emissions from $\mathrm{GaAs}(1 \times 2)$-Sn (top) and pure GaAs- $c(8 \times 2)$ (center) after air exposure and annealing in vacuum, and from pure GaAs- $c(8 \times 2)$ before air exposure (bottom). (b) O $1 s$ emissions from $\operatorname{GaAs}(1 \times 2)$-Sn (bottom and top) and GaAs-c $(8 \times 2)$ (center) after air exposure and annealing at different temperatures. (c) LEED $(120 \mathrm{eV})$ from $\operatorname{GaAs}(1 \times 2)$-Sn after $1 \mathrm{~h}$ air exposure and $0.5 \mathrm{~h}$ annealing at $550{ }^{\circ} \mathrm{C}$, showing a clear $(1 \times 2)$ pattern. (d) LEED $(57 \mathrm{eV})$ from InAs $(1 \times 2)$-Sn after $1 \mathrm{~h}$ air exposure and $0.5 \mathrm{~h}$ annealing at $450{ }^{\circ} \mathrm{C}$, showing a clear $(1 \times 2)$ pattern. (e) LEED $(55 \mathrm{eV})$ from $\operatorname{InAs}(1 \times 2)$-Sn after $1 \mathrm{~h}$ air exposure and $0.5 \mathrm{~h}$ annealing at $470{ }^{\circ} \mathrm{C}$, showing a clear $c(8 \times 2) /(4 \times 2)$ pattern.

broadening or band bending, consistently with the absence of $\mathrm{O} 1 \mathrm{~s}$ emission [Fig. 1(b) bottom]. Moreover, the GaAs $(1 \times 2)$-Sn surface exhibited a sharp LEED [Fig. 1(c)] after air exposure and heating. Even after air exposure for one week, the $\mathrm{GaAs}(1 \times 2)$-Sn produced a clear $(1 \times 2)$ LEED after heating to $580{ }^{\circ} \mathrm{C}$; at the same time the $\mathrm{Ga} 3 d$ peak did not include the oxide emission. The LEED from samples exposed to air for a week was of course not as sharp as in Fig. 1(c) but the quality of the pattern improved with prolonged heating. It is important to note that after air exposure but before any heating, both the $\mathrm{GaAs}(1 \times 2)-\mathrm{Sn}$ and the pure GaAs- $c(8 \times 2)$ samples exhibited a poor $(1 \times 1)$ LEED and $\mathrm{Ga}$ oxide emissions. The $\operatorname{InAs}(1 \times 2)$-Sn behaves in the same way as the $\operatorname{GaAs}(1 \times 2)$-Sn after air exposure but it has some unexpected properties as follows: Sn re-evaporated from the InAs more easily than from the GaAs since heating to $470{ }^{\circ} \mathrm{C}$ removed $\mathrm{Sn}$ from the InAs and produced the pure InAs- $c(8 \times 2)$ surface [Fig. 1(e)] while the $(1 \times 2)$-Sn remained on the $\mathrm{GaAs}$ at least up to $600{ }^{\circ} \mathrm{C}$. Our preliminary tests show that $\operatorname{InSb}(100)-\mathrm{Sn}$ system behaves like the InAs(100)-Sn.

Figure 2 shows high-resolution photoemission spectra from $\mathrm{InAs}(1 \times 2)$-Sn before and after air exposures and also after heating the air-exposed samples. The same measurements of $\operatorname{GaAs}(1 \times 2)-\mathrm{Sn}$ reveal the behavior (not shown) similar to that in Fig. 2. The high binding energy (BE) components $S^{*}$ clearly indicate that the GaAs and InAs substrate surfaces are oxidized during air exposure. ${ }^{24,25}$ It also appears that As oxides are the first oxides to be removed by heating. The Sn $4 d$ spectra also reveal many surface components $\mathrm{S}^{*}$ and $\mathrm{S}^{* *}$ at higher $\mathrm{BE}$ than the $\operatorname{InAs}(1 \times 2)-\mathrm{Sn}$ peaks; this agrees closely with previous reports, showing similar peaks between 1.5 and $2.5 \mathrm{eV}$ measured from $\mathrm{SnO}$ and $\mathrm{SnO}_{2}$ compounds. $^{26}$

To interpret the experimental findings, $a b$ initio calculations were performed using the projector augmented wave method as implemented in the Vienna $a b$ initio Simulation Package. $^{27,28}$ The plane wave cutoff energy was set at 400 $\mathrm{eV}$. The computation procedure is described elsewhere. ${ }^{20,29}$ The adsorption of an oxygen atom was simulated for different surfaces, including $c(8 \times 2), \beta(2 \times 4)$, and Sn-induced $(1 \times 2)$. Several relevant surfaces were considered to rule out the possibility that the results were dependent on the surface chosen or on the position of the oxygen atom. One substitutional $\mathrm{Sn}$ atom was inserted into the $c(8 \times 2)$ and $\beta(2 \times 4)$ reconstructions while on the $\mathrm{Sn}$-stabilized surface $\mathrm{Sn}$ coverage varied between 0.125 and $0.625 \mathrm{ML}$. The Ga and As atoms in the first and second layers of GaAs-Sn were gradually substituted by equivalent $\mathrm{Sn}$ atoms, and several different relatively stable adsorption sites were considered. Local Sn clustering was also tested. The $(4 \times 2)$ unit cells adopted enabled the comparison of similar oxygen adsorption sites with different nearest-neighbor environments. The total energy results show consistently, regardless of surface or Sn coverage, that by replacing a $\mathrm{Ga} / \mathrm{In}$ (As) atom with a $\mathrm{Sn}$ atom the adsorption/desorption energy of a neighboring $\mathrm{O}$ atom is decreased (increased) by $0.1-0.8 \mathrm{eV}$ (most of the shifts are within $0.4 \mathrm{eV}$ ); in other words, it is easier (more difficult) to desorb oxygen. The tendency observed is thus probably also valid on a more or less disordered surface which is relevant, because surface is reconstructed upon oxidation. The results are in agreement with the experimental observation according to which As oxides are the first oxides to be removed by heating. If some of the $\mathrm{Ga} / \mathrm{In}$ atoms are replaced by $\mathrm{Sn}$ atoms, the surface tends to desorb oxygen more easily. We recall that the GaAs surface is crystallized in the $\zeta-c(8$ $\times 2$ )-structure ${ }^{29}$ in Ga-rich conditions at $0 \mathrm{~K}$ (although under finite temperatures there are also לa-type areas on the surface ${ }^{30,31}$ ), and the number of Ga atoms in the surface layer is slightly reduced in the Sn-induced phase transition to the $(1 \times 2)$ reconstruction. The absolute value of the adsorption energy depends strongly on the adsorption position (scatter in adsorption energy is more than $1 \mathrm{eV}$ ), which is a common result for all surfaces considered. 

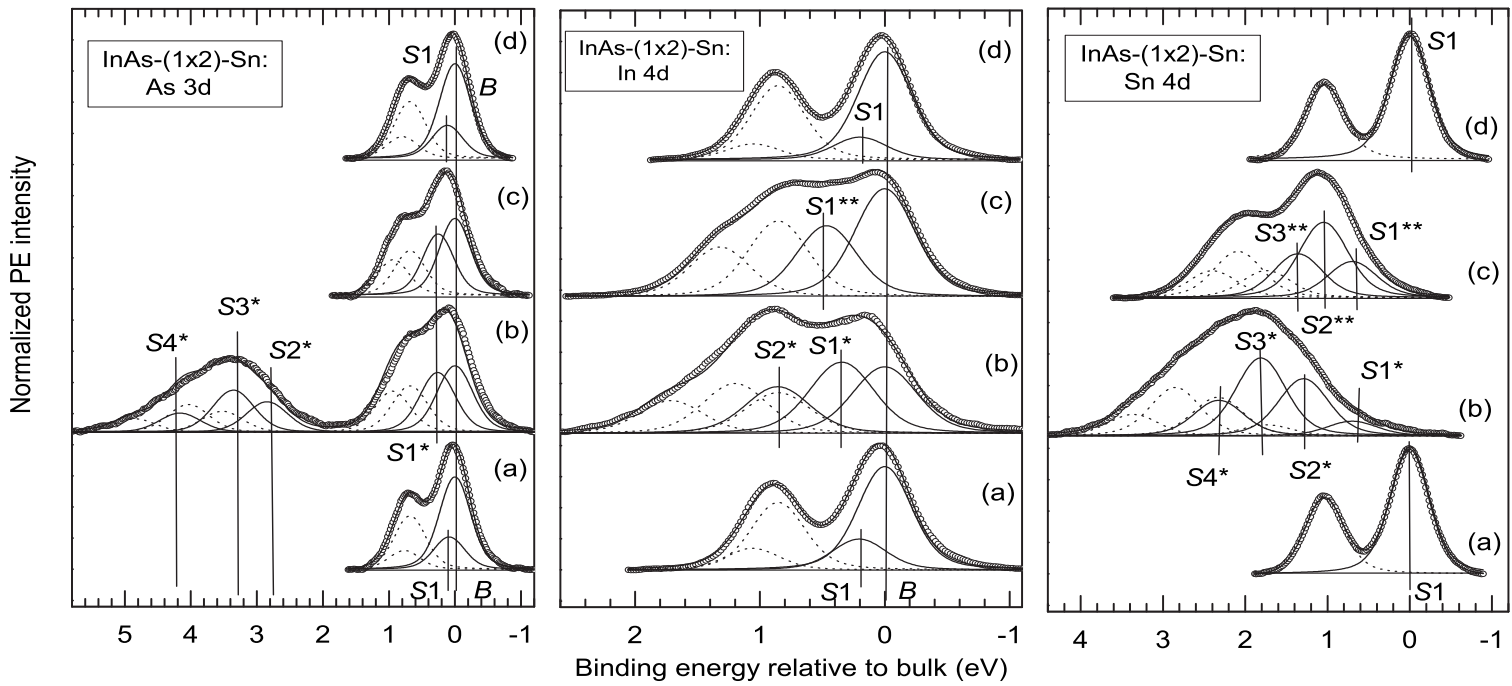

FIG. 2. As $3 d$, In $4 d$, and Sn $4 d$ photoemissions from InAs(1 $\times 2)$-Sn (a) before air exposure, (b) after air exposure, and [(c) and (d)] after heating of the air-exposed sample at $420{ }^{\circ} \mathrm{C}$ and $450{ }^{\circ} \mathrm{C}$, respectively. The spectra were fitted with the minimum number of components. $B$ represents InAs bulk components; different $S$ components are surface-related. Sn $4 d$ spectra were aligned with In $4 d$ bulk components.

As the Sn coverage on the $(1 \times 2)$ surface is only around $0.5 \mathrm{ML}$, and not many $\mathrm{Ga}(\mathrm{In})$ atoms are replaced with $\mathrm{Sn}$ atoms in the Sn-induced reconstruction change, we suggest that the Sn-induced surface structure/composition change has a great effect on oxygen desorption. (Most favorable adsorption sites are within the surface layers.) Sn adsorption induces the $(1 \times 2)$ reconstruction, in which there are only four $\mathrm{Ga}$ (In) atoms in the surface layer and no Ga (In) atoms in the second layer of the $(4 \times 2)$ surface area. On the other hand, the $\zeta-c(8 \times 2)[\zeta \mathrm{a}-c(8 \times 2)]$ unit cell of the III-V compound comprises six (eight) $\mathrm{Ga}$ (In) atoms in the more dense III-V mixed surface layer and eight $\mathrm{Ga}$ (In) atoms in the second layer of the $(4 \times 2)$ surface area. Thus there will be much more relatively strong $\mathrm{Ga}-\mathrm{O}$ bonds within the surface layers of pure GaAs upon oxidation compared to $\mathrm{Sn} / \mathrm{GaAs}$. There are also adsorption positions in the second layer of the $c(8 \times 2)$ structure which have a peculiarly large $\mathrm{BE}$, because in these positions the $\mathrm{O}$ atoms are bound only to group III atoms. In the $(1 \times 2)$ structure the most favorable oxygen position is on top of the $\mathrm{Ga}(\mathrm{In})$-Sn dimer in the surface layer, which may also facilitate oxygen desorption. The energy difference between the most favorable adsorption positions in the $(1 \times 2)$ and $\zeta-c(8 \times 2)[(1 \times 2)$ and $\zeta \mathrm{a}-c(8 \times 2)]$ reconstructions is about $0.2 \mathrm{eV}[0.5 \mathrm{eV}]$.

To recapitulate: the $(1 \times 2)$-Sn layer did not prevent substrate oxidation and the formation of an amorphous surface layer in air, but significantly improved the removal of surface oxides (enabled effective surface cleaning with heating of the samples). Our results agree with, and may also provide a partial explanation for, the beneficial effects of Sn on III$\mathrm{V}(100)$ surfaces; ${ }^{32-35}$ the deposition of an Sn prelayer in the epitaxial growth of GaAs effectively eliminates interfacial defects. ${ }^{32}$ Furthermore, an Sn submonolayer has been found to act as a surfactant in GaAs epitaxial growth. ${ }^{33} \mathrm{Sn}$ is also an $n$-type dopant of III-Vs, and Sn-terminated growth fronts have been found to affect bulk doping concentrations. ${ }^{35} \mathrm{Re}-$ cent studies ${ }^{20,36}$ have elucidated the atomic structures of the $(1 \times 2)$-Sn surfaces.
We are grateful to $\mathrm{H}$. Ollila and the MAX-laboratory staff for technical assistance. The support of the Transnational Access to Research Infrastructure Program (TARI) is acknowledged. This work has also been supported by Academy of Finland Grant No. 122743 (P.L.), by the National Graduate School in Materials Physics (J.L.), and by the Solar III-V Project (Dnro: 3120/31/08). The calculations were performed at the Finnish IT Center for Science.

\footnotetext{
${ }^{1}$ Fundamentals of III-V Semiconductor MOSFETS, edited by S. Oktyabrsky and P. D. Ye (Springer, New York, 2010).

${ }^{2}$ C. J. Sandroff et al., Appl. Phys. Lett. 51, 33 (1987).

${ }^{3}$ M. Hong et al., Science 283, 1897 (1999).

${ }^{4}$ H. Hasegawa et al., J. Vac. Sci. Technol. B 18, 2100 (2000).

${ }^{5}$ N. Negoro et al., J. Vac. Sci. Technol. B 21, 1945 (2003).

${ }^{6}$ Y. Liang et al., Appl. Phys. Lett. 85, 1217 (2004).

${ }^{7}$ R. F. Klie et al., Appl. Phys. Lett. 87, 143106 (2005).

${ }^{8}$ S. Koveshnikov et al., Appl. Phys. Lett. 88, 022106 (2006).

${ }^{9}$ C. L. Hinkle et al., Appl. Phys. Lett. 92, 071901 (2008).

${ }^{10}$ E. O'Connor et al., Appl. Phys. Lett. 92, 022902 (2008).

${ }^{11}$ B. Shin et al., Appl. Phys. Lett. 93, 052911 (2008).

${ }^{12}$ P. D. Ye, J. Vac. Sci. Technol. A 26, 697 (2008).

${ }^{13}$ T. Anan et al., Appl. Phys. Lett. 63, 1047 (1993).

${ }^{14}$ R. A. McKee et al., Phys. Rev. Lett. 81, 3014 (1998).

${ }^{15}$ T. Ohta et al., Phys. Rev. Lett. 94, 116102 (2005).

${ }^{16}$ D. A. Ricci et al., Phys. Rev. Lett. 95, 266101 (2005).

${ }^{17}$ E. Lu et al., Phys. Rev. Lett. 97, 146101 (2006).

${ }^{18}$ J. W. Reiner et al., Phys. Rev. Lett. 101, 105503 (2008).

${ }^{19}$ Y. Segal et al., Phys. Rev. Lett. 102, 116101 (2009).

${ }^{20}$ J. J. K. Lång et al., Surf. Sci. 605, 883 (2011).

${ }^{21}$ P. Laukkanen et al., Phys. Rev. B 72, 045321 (2005).

${ }^{22}$ P. Laukkanen et al., Appl. Phys. Lett. 90, 082101 (2007).

${ }^{23}$ P. Laukkanen et al., J. Electron Spectrosc. Relat. Phenom. 177, 52 (2010).

${ }^{24}$ C. L. Hinkle et al., Appl. Phys. Lett. 94, 162101 (2009).

${ }^{25}$ D. C. Suh et al., Appl. Phys. Lett. 96, 142112 (2010).

${ }^{26}$ M. Batzill and U. Diebold, Prog. Surf. Sci. 79, 47 (2005).

${ }^{27}$ G. Kresse and J. Hafner, Phys. Rev. B 47, 558 (1993).

${ }^{28}$ G. Kresse and J. Furthmüller, Phys. Rev. B 54, 11169 (1996).

${ }^{29}$ J. J. K. Lång et al., Phys. Rev. B 81, 245305 (2010).

${ }^{30}$ C. Kumpf et al., Phys. Rev. Lett. 86, 3586 (2001)

${ }^{31}$ A. Ohtake et al., Phys. Rev. B 65, 233311 (2002)

${ }^{32}$ C.-A. Chang et al., Appl. Phys. Lett. 39, 229 (1981).

${ }^{33}$ G. S. Petrich et al., Appl. Phys. Lett. 61, 162 (1992).

${ }^{34}$ M. B. Panish et al., Appl. Phys. Lett. 56, 2301 (1990).

${ }^{35}$ J. J. Harris et al., Surf. Sci. 103, L90 (1981).

${ }^{36}$ D. Usanmaz et al., Surf. Sci. 603, 2683 (2009).
} 